\title{
Affordability Engineering: Bridging the Gap between Design and Cost
}

\author{
J.D. Reeves ${ }^{1}$ \\ NASA Langley Research Center, Hampton, VA, 23681 \\ Dominic DePasquale ${ }^{2}$ \\ SpaceWorks Engineering, Inc., Washington, DC, 20006 \\ and \\ Evan $\operatorname{Lim}^{3}$ \\ Science Applications International Corporation (SAIC), Sunnyvale, CA, 94087
}

\begin{abstract}
Affordability is a commonly used term that takes on numerous meanings depending on the context used. Within conceptual design of complex systems, the term generally implies comparisons between expected costs and expected resources. This characterization is largely correct, but does not convey the many nuances and considerations that are frequently misunderstood and underappreciated. In the most fundamental sense, affordability and cost directly relate to engineering and programmatic decisions made throughout development programs. Systems engineering texts point out that there is a temporal aspect to this relationship, for decisions made earlier in a program dictate design implications much more so than those made during latter phases. This paper explores affordability engineering and its many sub-disciplines by discussing how it can be considered an additional engineering discipline to be balanced throughout the systems engineering and systems analysis processes. Example methods of multidisciplinary design analysis with affordability as a key driver will be discussed, as will example methods of data visualization, probabilistic analysis, and other ways of relating design decisions to affordability results.
\end{abstract}

$\begin{array}{ll} & \\ \text { CAIV } & =\text { Cost as an Independent Variable } \\ \text { CDTC } & =\text { Conceptual Design to Cost } \\ \text { CER } & =\text { Cost Estimating Relationship } \\ \text { DDT\&E } & =\text { Design, Development, Test, \& Evaluation } \\ \text { DOE } & =\text { Design of Experiments } \\ \text { DTC } & =\text { Design to Cost } \\ \text { ECLS } & =\text { Environmental Control \& Life Support } \\ \text { FOM } & =\text { Figure of Merit } \\ \text { I-RaCM } & =\text { Integrated Risk and Cost Model } \\ \text { MDO } & =\text { Multidisciplinary Optimization } \\ \text { NAFCOM } & =\text { NASA Air Force Cost Model } \\ \text { NEO } & =\text { Near Earth Object } \\ \text { TFU } & =\text { Theoretical First Unit } \\ \text { WBS } & =\text { Work Breakdown Structure }\end{array}$

\footnotetext{
${ }^{1}$ Life Cycle Engineer, Space Mission Analysis Branch/Systems Analysis and Concepts Directorate, AIAA Member.

${ }^{2}$ Director, Engineering Economics Group, AIAA Member.

${ }^{3}$ Consultant.
} 


\section{Introduction}

A FFORDABILITY is a term often used throughout engineering domains to convey a comparison between expected costs and expected available resources. Expected costs are generally predicted design and production costs based on a variety of estimating methods, while available resources typically take the form of expected budgets allocated to the task at hand. There are many subtleties and perspective differences involved such as whether a design and development organization is government or commercial, whether budgets or cost targets are hard constraints, and to what degree the results of affordability comparisons should influence the design itself. The scope of what is being assessed can also vary, for affordability comparisons are needed for system acquisition processes of all sizes; from the smallest information technology system procurement to an entire exploration architecture consisting of numerous hardware and software systems. Affordability is often considered a natural extension, and general characterization of, cost estimating processes and results. Cost estimating itself is often considered part of the engineering process, but too often is performed and considered late in the design process with minimal influence on the designs themselves.

However, both affordability and cost analysis are engineering activities, and represent a significant portion of proper systems engineering. Instead of cost and affordability being analysis activities at the end of the conceptual design process, they should be considered iterative and should influence design decisions from the beginning. To that end the concept of affordability engineering is introduced.

This paper consists of several sections that introduce, discuss, and demonstrate affordability engineering as a systems engineering principle. Section II provides a discussion of nomenclature and popular affordability and cost paradigms such as Cost as an Independent Variable (CAIV). Section III furthers the concept of affordability engineering by discussing various systems engineering and systems analysis principles that relate affordability engineering to the larger design process. This section also provides a proposed framework that breaks affordability engineering into its constituent sub-disciplines, each with their own challenges, methods, and considerations. Section IV introduces a fictional multi-system architecture design example to demonstrate the various affordability engineering methods and principles. Section V concludes the paper by summarizing the authors' proposed concept of affordability engineering.

\section{Nomenclature and Strategies}

To understand the subtleties between the concepts of cost and affordability several definitions should be understood. First, both cost and affordability are far more complex than simply dollars and budgets. A 1971 text from Fisher ${ }^{1}$ describes cost by stating "costs are consequences", and that "it is in alternatives, it is in foregone opportunities, that the real meaning of "cost' must always be found". Similarly, "an estimate of the cost of any such choice or decision is an estimate of the benefits that could otherwise have been obtained". Although these definitions at first seem to be aimed at net present value considerations between competing alternatives, they do drive at a more fundamental principle that is a major concern when assessing large and complex systems. Every dollar, man-hour, or other resource committed to performing one task is inherently not available to concurrently perform some alternative second task. When an organization, especially one as large as the U.S. Government, decides to pursue and invest in project $\mathrm{X}$, they are forgoing the time, resources, and money that could have been spent on project Y. Within NASA, annual budgets are typically established at the Mission Directorate level (or lower), so each directorate level decision maker only has a finite amount of funds and personnel to support their entire portfolio. Since cost estimates generated today often summarize expected expenditures in future years, such resource allocation decisions can have long term implications. The balancing of these implications and trade-offs, and how they relate to value gained from the investments themselves, is where cost and cost estimating become engineering disciplines unto themselves.

Affordability is somewhat tougher to define since it is often simply thought of as a comparison between costs and budgets. The NASA Cost Estimating Handbook $^{2}$ defines affordability as "the engineering process or management discipline which assures the final system, program, project, product, or service can be delivered (or owned, operated, developed, and produced) at a cost which meets previously-established funding (or best value) constraints while still meeting all approved requirements (or standards, needs, and specifications)". This definition summarizes the concept of comparing estimates to established funding levels, but does not capture aspects that define affordability as an engineering discipline. For example, not all design alternatives carry the same available budget since they could provide varying levels of value returned, which could lead to a reassessment of the amount of money available to spend. Overall resources may be constrained, but decision makers perpetually have to balance investment decisions within a portfolio, so individual allocations to various areas can always been traded. CAIV and Design to Cost (DTC) strategies exist (and are discussed below), but do not always carry the full understanding of 
what affordability engineering entails. Another consideration is the certainty of the overall amount of resources/funds available. Almost never is there a static budget assumption that remains constant throughout the full life of a project, for available funds are fluid and carry their own set of risks. From an engineering perspective, a design that truly meets stated requirements with an appropriate level of confidence includes margins of all sorts in order to be robust and flexible. Only by understanding both the expected and unexpected behavior of available funds, inclusive of various threats and liens that may exist, can an engineering team properly include cost and affordability engineering considerations within systems engineering and systems analysis processes. Only by taking such threats and liens into account can the integrated design be considered robust.

CAIV is a strategy that is well documented and used with success throughout numerous organizations. Brady ${ }^{3}$ describes the process as "making schedule and performance requirements functions of cost, which then becomes an independent variable in the system tradeoff equations. Analyses are performed to determine feasible variations in performance and schedule parameters while cost remains constant". This strategy is an improvement beyond older practices of ignoring cost and affordability ramifications until design is complete, and takes steps towards emphasizing the importance of cost and affordability within the design process. However this strategy comes with some challenges and shortfalls.

First, CAIV is difficult to implement within actual conceptual design programs for complex and integrated systems. This is largely because complex systems such as manned space systems are extremely difficult to close from a performance standpoint, and often rely on assumptions of advanced technology infusion, optimistic mass and power margin assumptions, and other uncertainties associated with challenging design requirements. In many applications of CAIV, the alternative tradeoff equations that Brady mentions do not involve a feasible performance solution space at all when holding cost constant. Relaxing design requirements is a logical and responsible step to open constrained solution spaces, but often times some requirements are so firm, such as direction to deploy a system by a fixed date, that no amount of requirements relaxation would result in a worthwhile design. The next step is often to relax schedules until enough funding becomes available, but this strategy does not address the primary problem. Only by striving to find reductions in expected costs based on design and programmatic decisions can costs be considered to be truly engineered.

A second issue with the standard implementation of CAIV is that is does not take into account interrelationships between multiple systems within a single architecture. Many cases, such as manned exploration architectures, require that requirements be traded and contrasted amongst various system elements that are highly dependent on each other. A mass increase in an in-space system clearly has implications on the delivery to LEO capability of its supporting launch vehicle. If CAIV suggests that performance and schedule requirements be traded while cost is held firm for individual systems within a multi-system architecture, how can cost ever be engineered to enable a completely optimal architecture solution? A solution to this issue may be a deeper investigation and explanation as to what is meant by CAIV, and how it can be applied to a multi-system architecture.

A similar technique is commonly known as the Design to Cost method of stating target cost estimates for various systems, subsystems, and/or components, and then striving as a team to engineer the system such that it results in a cost below the stated targets. Hari, Shoval, and Kasser ${ }^{4}$ suggest that because traditional DTC requires knowledge of "the cost of the actual components to be used in the system", that "DTC is not suitable for the use in the conceptual or preliminary design phases". However, their proposal of Conceptual Design to Cost (CDTC) builds upon traditional DTC by showing that focused cost engineering activities should target only the key areas that drive overall cost, as opposed to concerning oneself with cost estimating every available detail. This strategy builds upon Pareto principles of systems engineering and reinforces the strategy of performing key affordability engineering sensitivity trades to identify the items that actually drive the design. Such sensitivity analyses complement the multisystem architecture aspect of some design problems since top-level architecture sensitivity results can flow down and inform lower level analysis by indicating which areas (system, subsystem, or component) should be addressed with further sensitivity analyses. Only by achieving this level of understanding will engineers identify which areas to focus design trades intended to reduce expected costs and schedules.

Another consideration is that expected costs and schedules may be less dependent on technical designs than often thought. Every organization has significant "standing armies" of engineers, managers, and support personnel of all sorts. When assigned to work certain projects these personnel tend to continue to support that effort regardless of how often systems are actually deployed. To a large degree, the actual activities and organization constructs that support an acquisition activity may have far greater cost and schedule ramifications than system mass, power requirements, or other requirement-related characteristics. Dean ${ }^{5}$ characterizes this by stating that "cost is generated by an action upon an object", which can be fractalized by considering that a project supports (or genopersists per Dean) a system, project management supports a project, an organization supports project management, and so forth. The inherent "cost drivers are the difficulty of the process, the complexity of the object, and the size of the object". 
This poses a problem for engineering activities in the traditional sense, for it is difficult to address these concerns within conceptual studies despite the fact that they carry very real implications. Proper affordability engineering analysis requires that this be taken into account to the extent possible, whether it be to only influence uncertainty and risk discussions, or to be included within actual quantitative modeling.

To build upon these concepts a formal definition of affordability engineering is sought. Such a definition should reflect the many complexities and subtleties associated with cost and affordability in general. It should also clearly articulate that affordability analysis is separate but related to cost estimation and cost analysis, and that it should be effectively considered as much an engineering discipline as traditional physics-based design disciplines.

Affordability Engineering - "the interdisciplinary approach, engineering, and balancing of system design decisions, related cost/schedule estimates, and available resources to arrive at an integrated design that meets customer requirements with maximum utility. It involves the proper identification and characterization of engineering, programmatic, and other external cost drivers, acknowledges and incorporates related uncertainties and risks, and provides the mechanism and insight to understand the value gained and opportunities lost due to various design options."

\section{Affordability Engineering as an Engineering Principle}

Affordability considerations within conceptual design may be the single most important set of considerations throughout a system's lifecycle. Systems engineering texts routinely suggest that up to $70 \%$ of total system costs are decided during conceptual design. This behavior is illustrated in Figure 1 from the INCOSE Systems Engineering Handbook. ${ }^{6}$ Although conceptual studies take up only $8 \%$ of the total time of a systems' existence, up to $70 \%$ of costs are committed due to decisions made during this time. Continued management and tracking of affordability trends throughout later development, production, and operations are critical to keeping costs under control, but cannot alter these early decisions. Although design detail is sparse throughout this early phase, and many do suggest that cost estimating efforts without such detail is not value-added, it can be clearly seen that the ramifications of decisions made based on what data is available at that time are significant. To account for that, effort has to be made to

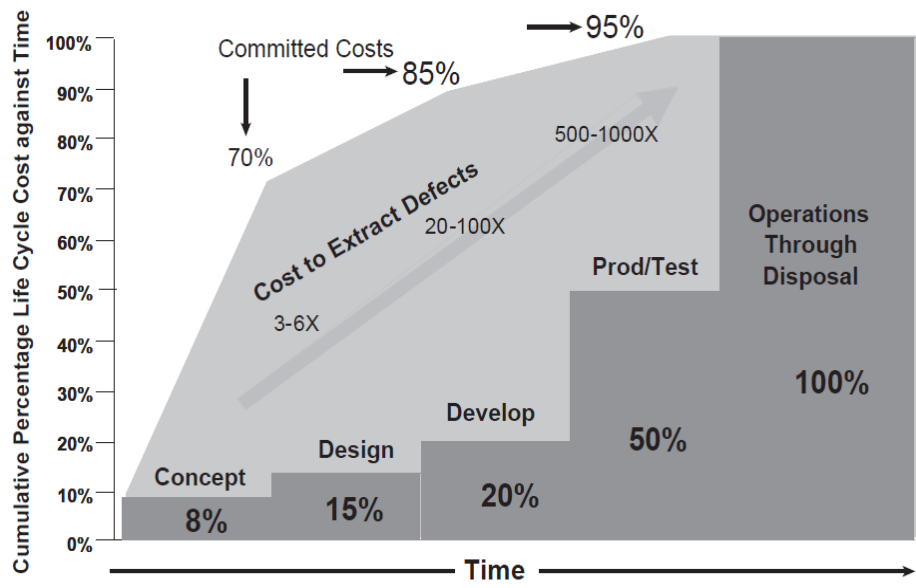

Figure 1. Committed life cycle cost against time (INCOSE). ${ }^{6}$ utilize to the maximum extent possible data that does exists, and to incorporate such data into formal affordability engineering practices to arrive at a clear understanding of long term implications of the various design decisions.

In order to further understand affordability engineering it is helpful to consider the numerous sub-disciplines that contribute to the discipline. Although a full list of methods, techniques, and skills required would require a separate dedicated effort, numerous general domains can be identified. The following seven affordability domains have been identified by the Systems Analysis and Concepts Directorate at the NASA Langley Research Center, and are shared here to help provide insight into what affordability engineering entails. It should be mentioned that these domains are not presented in any particular order or preference, for all should be considered equally. 


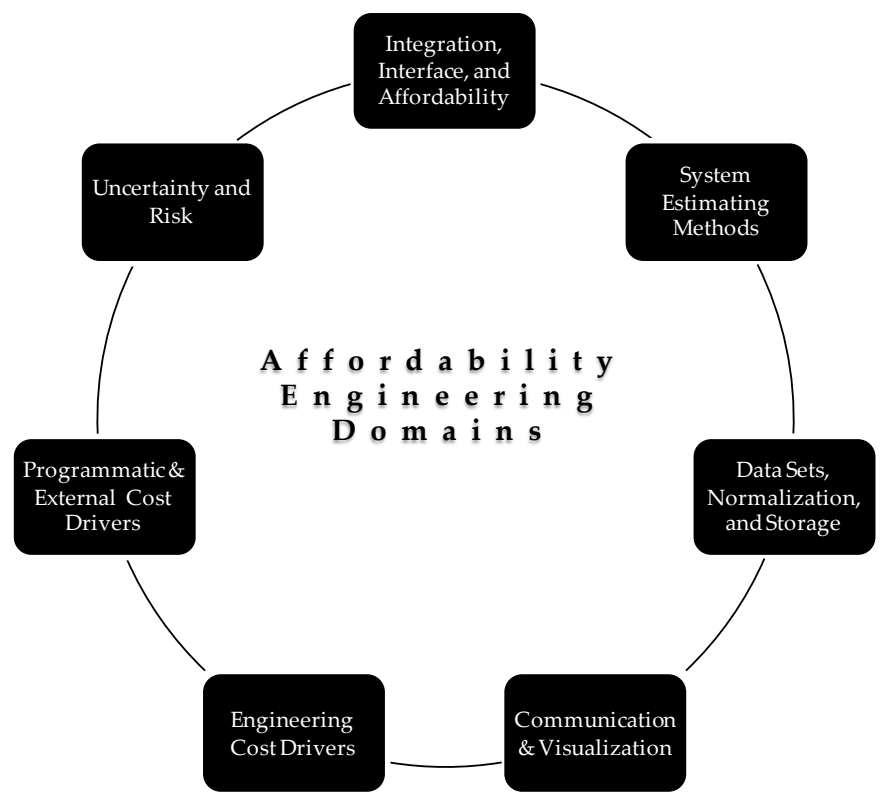

Figure 2. Proposed affordability engineering domain framework.

System Estimating Methods - in order for broad affordability to be assessed, the various hardware and software estimating methods and tools have to be sound. During conceptual design there is often not enough design detail available for detailed cost estimating, but general trends and analysis are still required. Being able to assess, even at a conceptual level, areas identified as key cost drivers (either system or architecture level) is essential to understanding affordability. This does not mean that every subsystem and component be estimated to a detailed level, but that enough analysis results are available for key engineering trades.

Data Sets, Normalization, and Storage - In order to support both cost and affordability engineering activities, appropriate data sets have to be collected, maintained, normalized, and stored in an accessible manner. In areas identified as significant cost drivers to both system and architecture costs, further data collection should be pursued to the extent required to provide adequate insight into expected behavior.

Communication \& Visualization - More so than possibly any other discipline, a large portion of affordability engineering is ensuring that resultant data be displayed and articulated in such a way that audiences, both internal and external, appreciate and understand various assumptions, key points, and underlying trends. Traditional sand charts are frequently used to display time-phased data relative to available budgets, but such plots should always be complemented by supporting sensitivity analysis results, additional trending diagrams, and other visualization methods needed to convey engineering trade considerations that involve cost and affordability.

Engineering Cost Drivers - One domain of affordability engineering that is commonly overlooked is the actual technical and performance drivers within systems that drive cost. Mass is commonly used as an indicator of expected cost trends, but every engineering discipline (e.g., propulsion, power, thermal, etc.) involves its own set of challenges, trends, and drivers. Instead of performance and sizing parameters simply being inputs into cost and affordability analysis, engineers working within the affordability realm should attempt to understand the individual engineering disciplines themselves to the extent needed to appreciate what drives system design. This aspect is at the core of why affordability engineering is simply multidisciplinary systems engineering in disguise.

Programmatic \& External Cost Drivers - Another domain essential to affordability engineering that is often underappreciated is programmatic and external influences and impacts to expected costs and budgets. Although political and similar behavior is very difficult to model and predict, an acknowledgement and 
general understanding of potential ramifications is always required. Some portion of risk and uncertainty discussions and considerations should certainly include an evaluation of these potential impacts.

Uncertainty and Risk - Uncertainties and related risks are prevalent throughout all aspects of design. Although formal confidence level analysis results are typically not required until latter design stages (when more design detail is available), an awareness of areas of high uncertainty and areas of potential risk is warranted throughout conceptual design. Formal methods and tools exist to probabilistically model joint cost and schedule confidence levels based on both parametric and bottoms-up estimating, so the use of these or similar tools should be pursued whenever possible even when designs are immature. Margins cannot account for the breadth of identified risks at this point in design, but an understanding of the more prominent risk areas should be pursued.

Integration, Interface, and Affordability - As mentioned in Section II, affordability engineering builds upon cost estimating and cost engineering in numerous ways. To some extent affordability engineering is the integration and analysis activity of numerous technical considerations, cost estimates, schedule estimates, budget uncertainties, and other related pieces of data. As will be demonstrated in Section IV, the integration phase includes key architecture and system-level sensitivity analyses that should be used to inform engineering trades.

The purpose of introducing these seven domains that define affordability engineering is to provide an understanding of the breath of sub-disciplines involved. It is the authors' suggestion that any organization seriously planning on improving overall affordability engineering capabilities, beyond traditional cost estimating, take steps to invest and improve upon each of these seven different areas. The scope of analysis may greatly differ between organizations, for one group may be looking to understand the ramifications of investing in a single instrument development activity while another may be weighing the pros and cons of an entire manned transportation architecture, but each of these domains will always be relevant. Numerous concepts and methods that support a number of these domains are illustrated in Section IV.

\section{Affordability Demonstration}

To illustrate the philosophy, principles, and components of affordability analysis, the authors have created a fictional story of space exploration set in the future. The story involves a very wealthy fisherman named Flash Gorton who wishes to visit Jupiter's moon Europa to go ice fishing for the exotic fish that have been discovered there. Flash Gorton's story and the parameters of the Europa Ice Fishing Program are described in more detail in the following section, and then referred to throughout the subsequent sections to illustrate various aspects of affordability analysis.

The process and sample results of the Europa Ice Fishing Program are exemplary of a typical affordability analysis. A roadmap for the affordability process is shown in Figure 3. Campaign definition and assessment (box 1) involves development of feasible sets of missions that meet customer requirements considering available technologies and operational concepts. Element costing (box 2) produces cost estimates for candidate system designs that are employed in the campaigns. The affordability activity (box 3) integrates the campaigns with the element costs and other cost sources to produce an integrated cost assessment and compare this against available budget and other resources. Risk assessment (box 4) in terms of system reliability defines likelihood of campaign operational success. Risk assessment in terms of affordability uncertainty is accomplished as part of the affordability activity (box 3) via probabilistic analysis. Finally, the relative merit of campaign possibilities is evaluated through Figure of Merit (FOM) analysis (box 5) to arrive at a program solution that is affordable and maximizes value. A feedback loop is essential to iterate on design variables, and sometimes constraints, in order to find a feasible solution. 


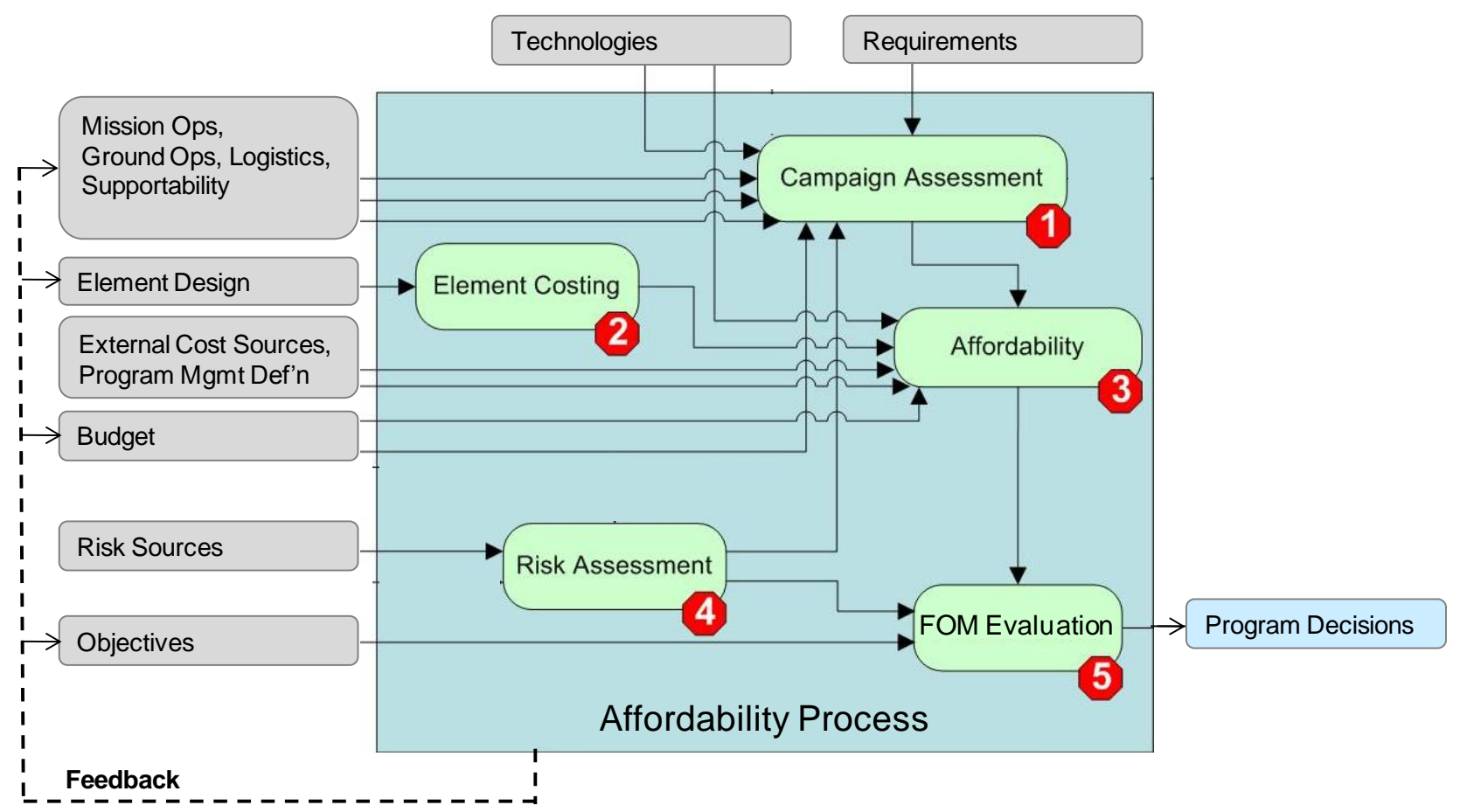

Figure 3. Affordability analysis interfaces and roadmap.

\section{A. Europa Ice Fishing Program}

Flash Gorton ran a highly successful fishing business. His keen knowledge and love of fishing, clever use of technology, hard work, and a little luck allowed him to grow a small fishing business into a global corporate juggernaut, and it made him very wealthy. In 2030, Flash Gorton's excitement about fishing reached all new levels when a robotic NASA lander discovered a sea teeming with exotic fish below the icy surface of Jupiter's moon Europa. He decided he must travel to Europa to go fishing, and dedicated himself and his personal assets to accomplishing that feat. Being the entrepreneur that he was, Flash furthermore figured he could return some of the fish to Earth and farm them for sale. Thus Flash envisioned a campaign of fishing missions to explore Europa and return the exotic fish to Earth.

Advances in materials and space propulsion in the 2020s had significantly improved in-space transportation, reducing the time of flight from Earth to Europa to just under two years as opposed to 6 or 8 years. Technology development would still be needed for multiple aspects of the Europa Ice Fishing Program including protection from the space environment during transit, long-term closed-loop life support, and ice fishing in the harsh environment of Europa. The Europa ice fishing missions would use existing Earth-to-orbit launch vehicles.

Flash Gorton anticipated conducting an extensive analysis of alternatives to assess the many possible mission architectures, hardware systems, technologies, and campaign approaches. Pre-Phase A studies would begin immediately in the year 2030, and the first crewed Europa landing would nominally occur in 2044, followed by one mission to Europa every two years after that.

Fortunately for Flash Gorton, NASA was willing to assist with design of the program in exchange for scientific data collected at Europa. Flash knew that the NASA engineers were capable of designing and building the systems necessary for the Europa Ice Fishing Program, but he had limited funds and thus needed to make sure that the design was affordable. Flash estimated that, through a combination of his savings and expected profits from his fishing business on Earth, the budget available for development of the Europa Ice Fishing Program is as shown in Table 1. 
Table 1. Available budget for Europa Ice Fishing Program (FY 2030 \$B).

\begin{tabular}{|c|c|c|c|c|c|c|c|c|c|c|c|}
\hline $\mathbf{2 0 3 0}$ & $\mathbf{2 0 3 1}$ & $\mathbf{2 0 3 2}$ & $\mathbf{2 0 3 3}$ & $\mathbf{2 0 3 4}$ & $\mathbf{2 0 3 5}$ & $\mathbf{2 0 3 6}$ & $\mathbf{2 0 3 7}$ & $\mathbf{2 0 3 8}$ & $\mathbf{2 0 3 9}$ & $\mathbf{2 0 4 0}$ \\
\hline 1 & 2 & 4 & 5 & 6.5 & 10.5 & 15 & 18 & 18 & 17.5 & 17.2 \\
\hline $\mathbf{2 0 4 1}$ & $\mathbf{2 0 4 2}$ & $\mathbf{2 0 4 3}$ & $\mathbf{2 0 4 4}$ & $\mathbf{2 0 4 5}$ & $\mathbf{2 0 4 6}$ & $\mathbf{2 0 4 7}$ & $\mathbf{2 0 4 8}$ & $\mathbf{2 0 4 9}$ & $\mathbf{2 0 5 0}$ & $\mathbf{2 0 5 1}$ \\
\hline 17 & 17 & 17 & 17 & 17 & 17 & 17 & 16 & 14 & 12 & 12 & TOTAL \\
\hline
\end{tabular}

There are any number of program alternatives that could satisfy the budget constraint and accomplish the overall objective of ice fishing on Europa. Some quantitative measures of goodness are needed to compare the value of the possible program solutions. Flash Gorton determined several metrics that were important to him, and from this defined Figures of Merit to evaluate the program alternatives. Table 2 summarizes the FOMs for the Europa Ice Fishing Program.

Table 2. Figures of merit for Europa Ice Fishing Program.

\begin{tabular}{|c|c|c|c|}
\hline FOM \# & Metric & Objective & Quantitative Measure \\
\hline FOM 1 & $\begin{array}{c}\text { Surface Stay } \\
\text { Time }\end{array}$ & Maximize time available for fishing & $\begin{array}{c}\text { Cumulative surface stay } \\
\text { days }\end{array}$ \\
\hline FOM 2 & Exploration & $\begin{array}{c}\text { Maximize fishing locations to improve } \\
\text { chances of catching different fish species }\end{array}$ & Number of sites visited \\
\hline FOM 3 & Cargo Returned & $\begin{array}{c}\text { Maximize cargo capacity for returning } \\
\text { fish to Earth }\end{array}$ & Mass of payload returned \\
\hline FOM 4 & Safety & Minimize the probability of harm to crew & Probability of Loss of Crew \\
\hline
\end{tabular}

\section{B. Cost Work Breakdown Structure}

Exploring the multidimensional trade space to identify solutions that satisfy program objectives and meet budget constraints is an incredibly complex problem. Given the complexity of the design space and the tremendous number of possible solutions, it is useful to have a common basis for comparison of multiple solutions. The Work Breakdown Structure (WBS) is a useful tool in this regard, providing an organizational structure for cataloging and comparing the many pieces of a program. A good cost WBS captures the complete costs of the program and provides sufficient detail to make meaningful comparisons between different possible program options. The Master Cost WBS shown in Figure 4 applies generically to many space exploration applications (e.g. Lunar, NEO, Mars) and provides a useful starting point for tailoring to the specific components of the architecture in question. Six levels of indenture have been defined for the Master Cost WBS as shown by the further breakdown of the Surface Systems domain area in Figure 4. The second level is the domain area, the third level the sub-domain area. The fourth level defines the organizational source of the system elements (e.g. government, commercial, international partner). The fifth level is the system or element level. For the case of Flash Gorton's Europa Ice Fishing Program, an example element might be a Pressurized Skimobile for transporting crew across the icy surface and exploring the moon. In the sixth level, the cost components of each system element are broken down into non-recurring (hardware and software), recurring (fixed and variable), and sustaining engineering costs. Together, these cost components cover the life cycle of the element. 


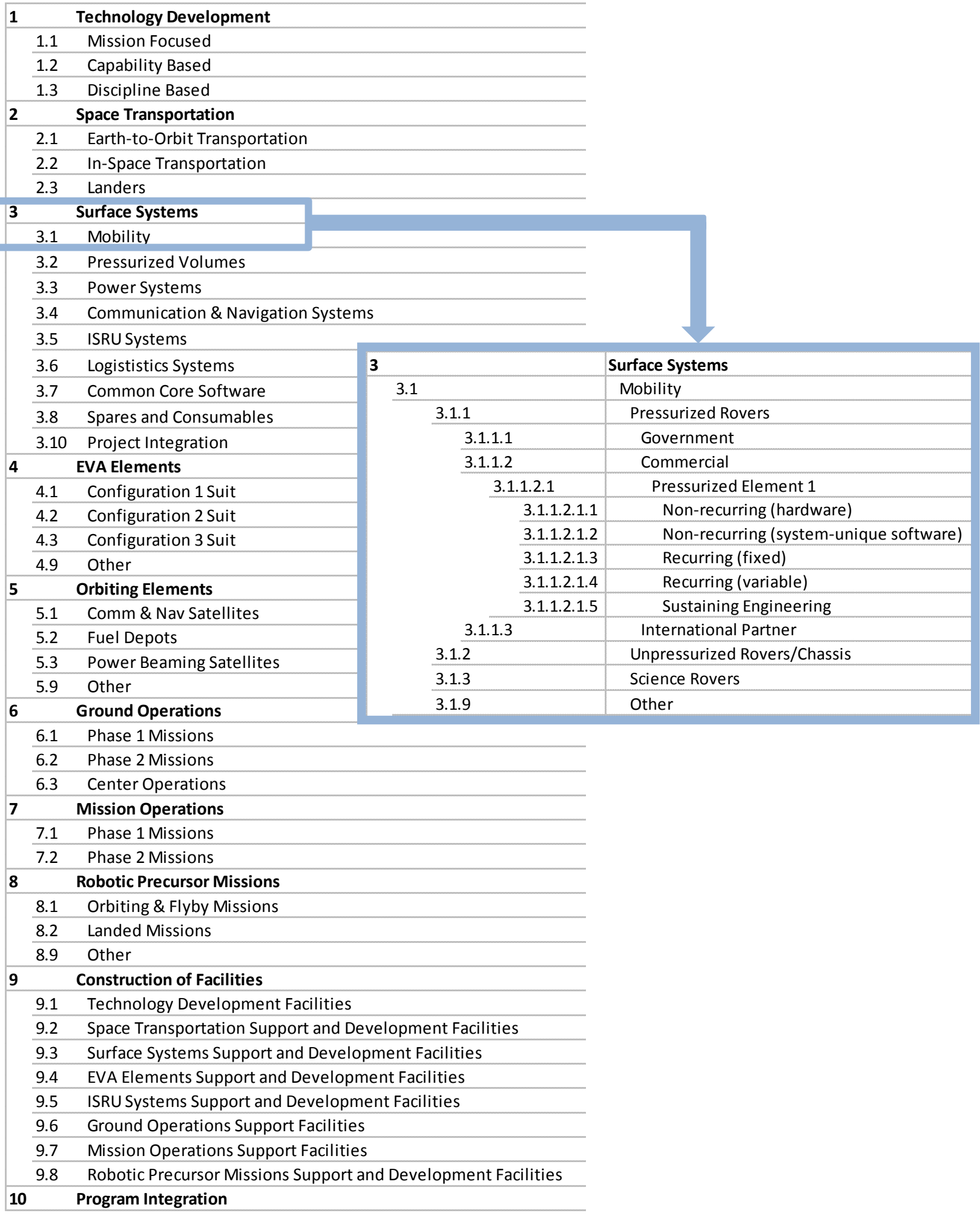

Figure 4. Master work breakdown structure. 


\section{Integrated Cost Estimates}

Program-level integrated cost estimates depict the complete cost picture and allow for affordability assessment. Integration commonly involves combining together a disparate set of systems-level cost estimates into a cohesive time-phased overall program cost estimate. There may be multiple elements and campaign manifests in the trade space that meet program objectives. The integration activity distributes element costs according to their development timelines and the campaign manifests. Individual element cost estimates are often in the form of Design, Development, Test, and Evaluation (DDT\&E) and Theoretical First Unit (TFU) costs that must be spread over the estimated duration of development and production respectively. A learning curve may be applied to production costs to model the effects of cost reduction as a result of industrial learning. The WBS serves as the structure for integrating and rolling up all the individual cost estimates, and helps to verify cost estimates for completeness and common assumptions.

An integrated cost estimate facilitates long-term program planning and allows for comparison to budget and other constraints. A sand chart is a convenient way to visualize the integrated cost estimate and its constituent parts. The Afford ${ }^{7}$ cost tool is capable of simply and quickly producing sand charts for any level of the integrated WBS. Figure 5 shows a sand chart for one of the candidate campaigns of Flash Gorton's Europa Ice Fishing program. In this example, the stratification of the sand is defined by the WBS Level 1 line items, with Space Transportation responsible for the largest portion of cost. Up front development costs are responsible for the peak cost in 2037, and cause a budget challenge as can be seen by comparing the sand to the red budget line. One possible solution for Flash Gorton to this budget shortfall in the early years is to delay development costs until later years where there is a budget surplus.

\section{LineItem Costs for Europa Ice Fishing Program}

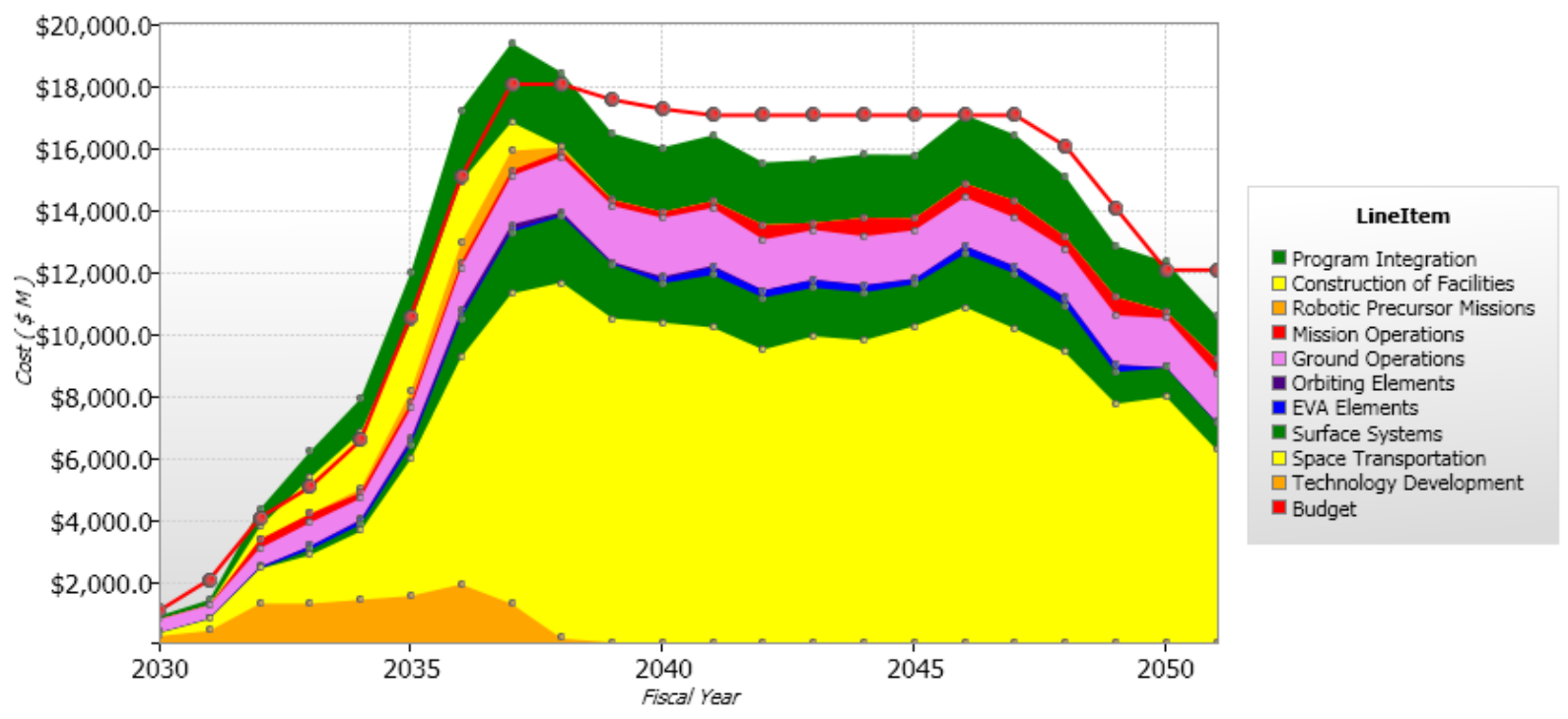

Figure 5. Sand chart for candidate campaign of Europa Ice Fishing Program. 
In the conceptual analysis of alternatives phase of a program, integrated cost estimates are useful for evaluating multiple program alternatives on the basis of required investment. Together with FOMs, the integrated cost estimates provide insight into the benefit versus cost of different program alternatives. One way to visually compare different program alternatives is the Outline sand chart, which shows the total cost profile of each alternative. It is also often useful to investigate the cumulative cost required to reach certain program milestones. This allows for an examination of campaign objectives across alternatives in the cost and schedule dimensions.
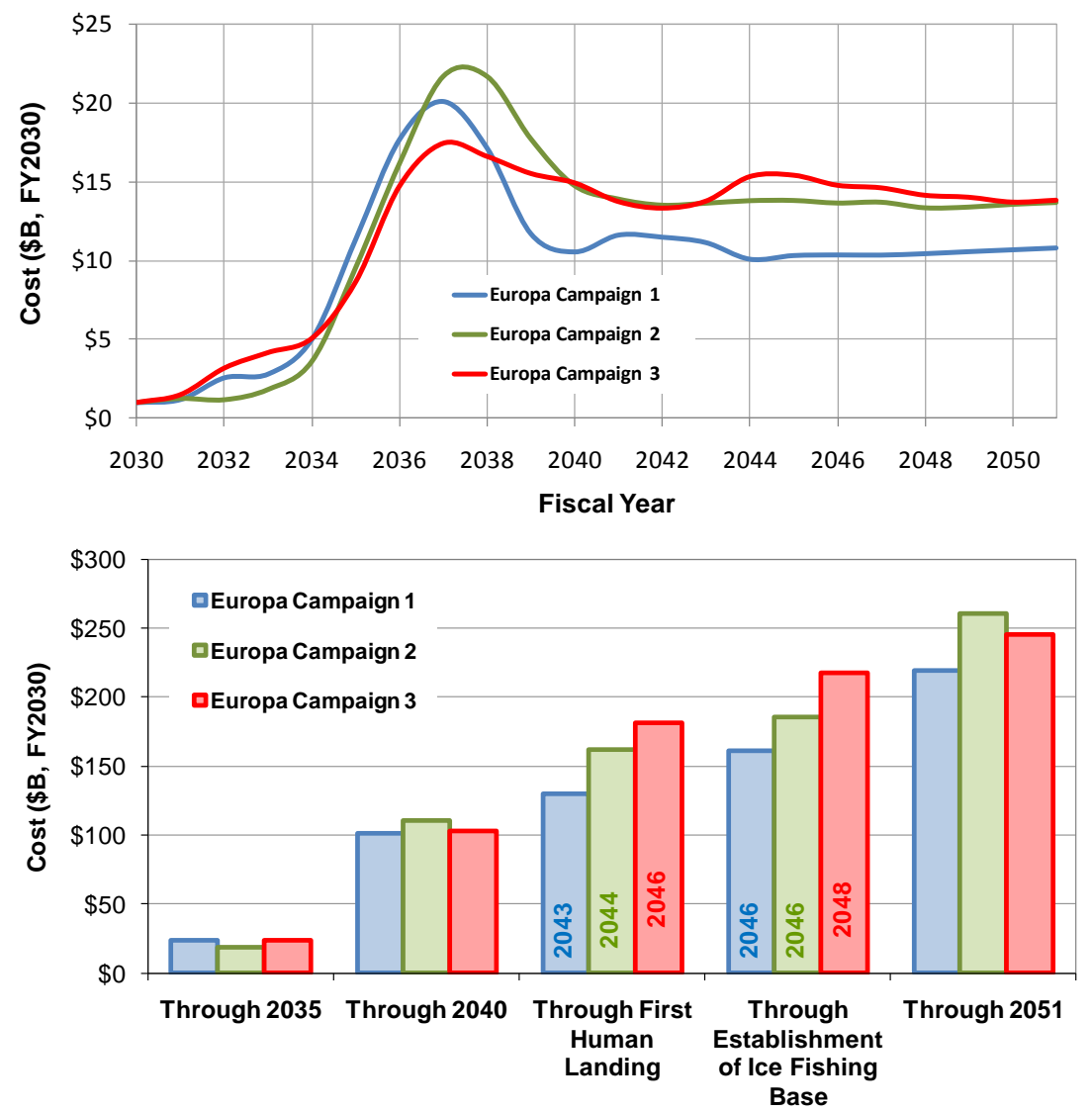

Figure 6. Europa Ice Fishing Program outline sand chart and cumulative cost bar chart showing cost to key milestones for several candidate campaigns.

\section{Multidisciplinary Design and Affordability Sensitivity Studies}

Multidisciplinary design optimization (MDO) frameworks enable rapid trade space exploration by integrating together multiple analysis tools. The Integrated Risk and Cost Model (I-RaCM) is one example of such a multidisciplinary model for concurrent assessment of program metrics such as cost, reliability, and operations. ${ }^{8}$ Performance disciplinary analysis tools can also be included in the MDO framework to couple performance design to cost estimating.

Implementing cost tools in a software integration and automation environment furthermore enables thorough sensitivity analysis and sophisticated uncertainty characterization. Probabilistic simulation, Design of Experiments (DOE) exploration, and optimization requiring hundreds to thousands of executions of cost and affordability tools can be automatically commanded. The ability to automatically generate large sets of data also enables metamodeling of individual tools and collections of linked tools. Response Surface Equations can be created and used to very rapidly conduct design trades.

Sensitivity analysis helps to identify cost drivers, better understand the workings of the tools, and ultimately arrive at more accurate cost estimates and improved affordability results. Sensitivity analysis also provides information for decision-making as the cost implications of design options are better understood. A sensitivity analysis main effects screening can be conducted at the architecture level as shown in Figure 7, or at the element level. In the Europa Ice Fishing program example shown in Figure 7, the architecture-level variables are the 
DDT\&E and TFU costs of the program elements. The Pareto, or "tornado," chart graphically displays results of a main effects screening which rank orders the input variables according to their contribution to variation (percent) in the response (total cost). For this example, one can conclude that for the set of input variables and their respective ranges examined, 21 percent of the variation in the response (campaign total cost) is attributable to the Ice Fishing Habitat DDT\&E cost.

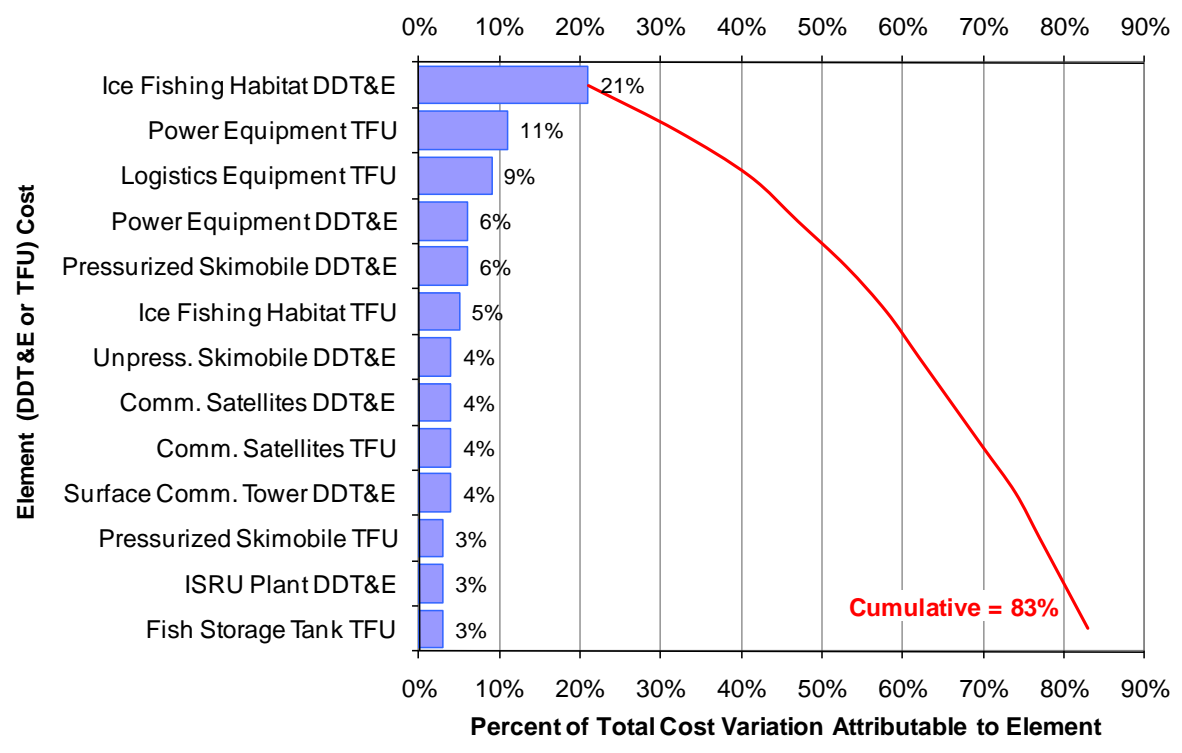

Figure 7. Europa ice fishing architecture surface system cost drivers.

A main effects screening conducted at the element level is shown in Figure 8. In this example, the element of interest was the Europa Ice Fishing Program Ice Fishing Habitat. The DDT\&E cost of this element was identified as the chief driver of the architecture surface systems, and is thus a good candidate for detailed investigation. The Ice Fishing Habitat cost estimate was completed using the industry standard NASA Air Force Cost Model (NAFCOM) developed by SAIC, so the variables of interest are the NAFCOM model input variables (the values in parenthesis indicating the baseline value associated with the estimate relative to the parameter's scale).

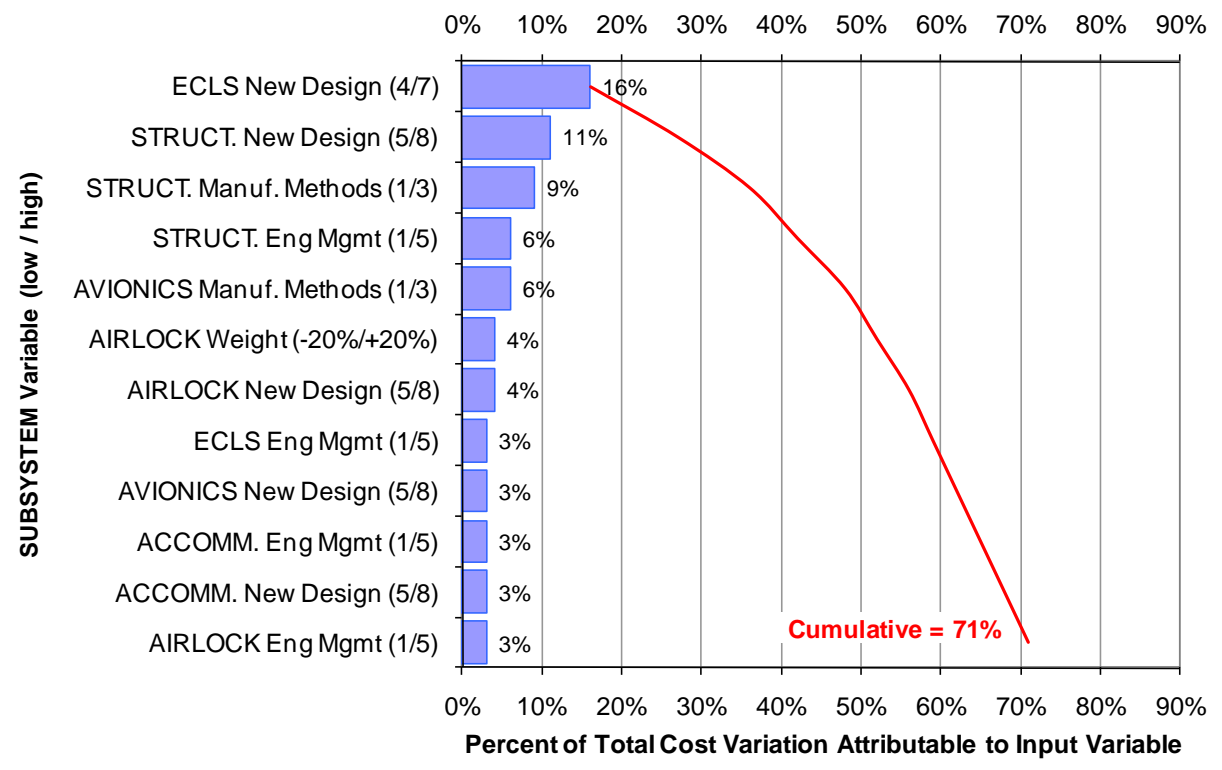

Figure 8. Europa Ice Fishing Habitat NAFCOM cost model drivers. 
Drilling down even deeper into an individual element cost estimate, parametric sweeps of individual input variables provide more detailed insight into the cost trends resulting from changing a single parameter. For the Europa Ice Fishing Program, the Ice Fishing Habitat was determined to be the leading contributor to Surface Systems cost variability, and the Environmental Control and Life Support (ECLS) subsystem New Design variable to be the leading contributor to the Ice Fishing Habitat variability. Further investigation of the ECLS subsystem is warranted to understand the sensitivity of cost model variables and evaluate their settings.

Figure 9 depicts four examples of parametric sweeps for variables of the ECLS subsystem. In order to aid users of the results, variables are categorized according to their effect on subsystem cost. Variables where cost differs by less than five percent between the baseline setting and the highest or lowest setting are categorized as having a "minimal effect" on subsystem cost. Those where the cost difference is between five and ten percent are categorized as having "some effect" on subsystem cost, and those where the cost difference is greater than ten percent are categorized as having a "major effect" on subsystem cost. Note that for the ECLS subsystem, as shown in the upper left graph in Figure 9, cost decreases as weight increases, an uncommon occurrence for the cost relationships of most subsystems.

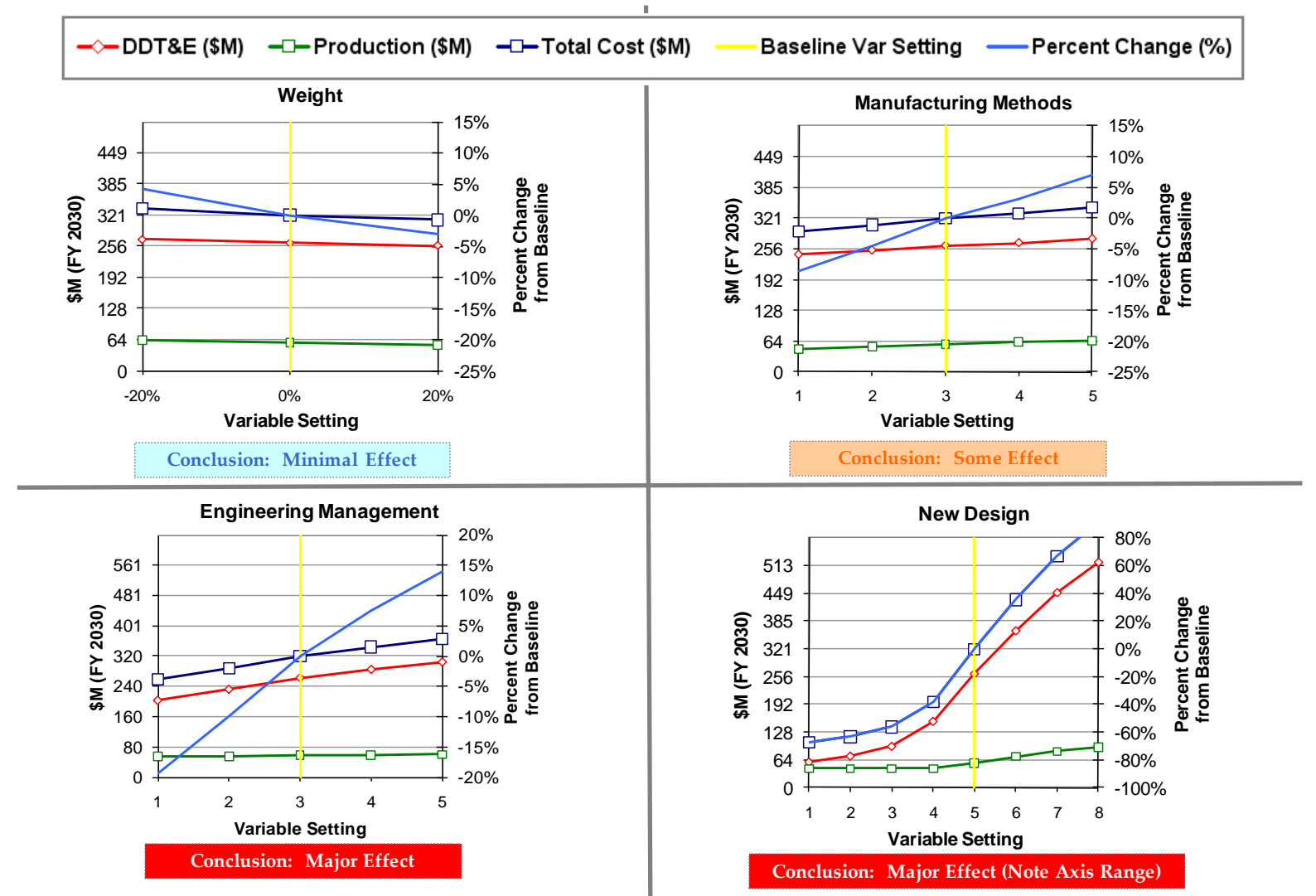

Figure 9. Europa Ice Fishing Habitat ECLS subsystem NAFCOM cost model parametric sweeps.

The Pareto and parametric sweep results shown in this section were produced using Phoenix Integration's ModelCenter ${ }^{\circledR}$ software integration environment and SpaceWorks Software's commercially available Remix software. ModelCenter® provides a user-friendly front-end and various built-in tools for integrating diverse software tools, performing trade studies, and collecting and analyzing data from a large number of executions of the integrated software tools. Remix allows the NAFCOM and Galorath Incorporated's SEER-H cost tools to interface with ModelCenter ${ }^{\circ}$, manages the transfer of inputs/outputs, and controls the cost tools at runtime. Remix exposes the NAFCOM and SEER-H inputs and outputs within ModelCenter ${ }^{\circledR}$ such that ModelCenter ${ }^{\circledR}$ 's built-in trade study tools can be used to perform parametric sweeps, design of experiments, Monte Carlo analysis, and other exploration studies. 


\section{E. Affordability Uncertainty}

At any point in the life of a program there will be uncertainty in the integrated cost estimate, and quantification of this uncertainty is critical to evaluating the affordability of the program. Sources of uncertainty include those arising from imperfect cost estimating, known discrete threats, and unknown threats that may exist but have not been conceived of by program management. Cost estimating uncertainty is quantifiable by stochastic treatment of the technical input variables and cost estimating relationship (CER) regression uncertainty. Threats may exist to the program plan, requirements, and budget that can be quantified in terms of probability of occurrence and monetary consequence. A probabilistic analysis of the integrated cost estimate results in a distribution of possible cost outcomes for the program. One popular software package for conducting such an analysis is the ACEIT suite of tools offered by Tecolote Research, Inc. Probabilistic "cost-risk" results for the Europa Ice Fishing Campaign 2 are given in Figure 10.

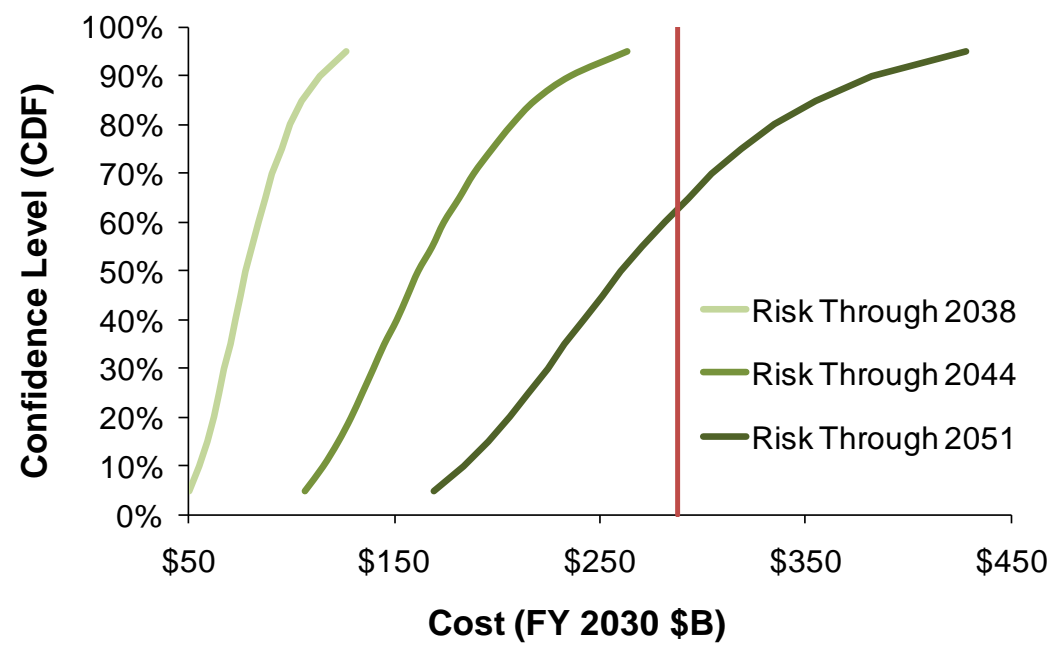

Figure 10. Europa Ice Fishing Program campaign 2 cost-risk results.

The cumulative distribution function "S-curves" shown in Figure 10 depict the probability of total program cost through three different years of the campaign. Though the S-curve appears to spread out over time, the relative uncertainty actually remains fairly constant. The relationship between percent change in cost and percent change in confidence level is the same in all years though the magnitude change in cost for a given percent change in confidence level increases with time. Comparing the total budget (\$287.7B in Table 1) for the Europa Ice Fishing Program to the third S-curve depicting cost-risk through 2051, it can be seen that the total budget is at the 63rd percentile of the expected cost outcomes determined by analysis. The acceptability of this budget confidence level depends on the amount of risk program management is willing to accept, but $63 \%$ is generally thought to be reasonable. As a program progresses, more risks are often identified resulting in increased uncertainty (S-curve spreads out), until the program matures to a point where risks are retired and design uncertainty decreases (S-curve narrows).

\section{F. Figure of Merit Evaluation}

A key aspect of affordability is the value achieved for a given expenditure of resources. Integrated cost results on their own do not provide insight into the benefit or value of different campaign options, but can be combined with FOMs to understand such value. Figure 11 shows plots of Flash Gorton's four FOMs for the Europa Ice Fishing Program versus cost of various campaign options. With Flash Gorton's budget of \$287.7 Billion (FY 2030), most of the campaign options shown here are feasible. If the budget was lesser, say \$250 Billion, many of the campaign options shown would not be feasible. Campaigns 1, 2, and 3 are highlighted. Campaign 1, for example offers the highest number of cumulative surface stay days at relatively low cost, but doesn't fare as well in the other FOMs. Campaign 2 is the most expensive campaign of the three, but does relatively well in all four FOMs. 

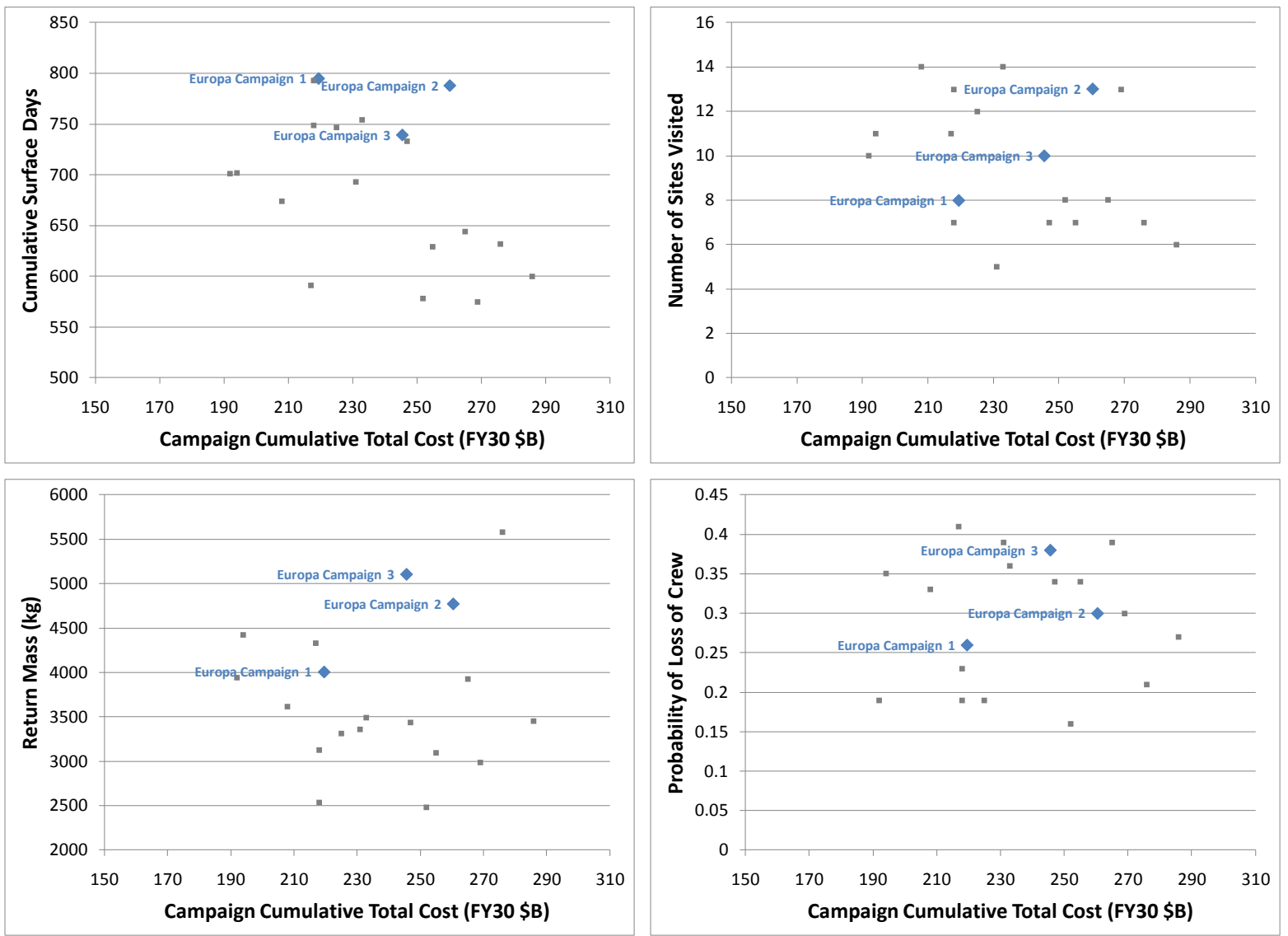

Figure 11. Europa Ice Fishing Program FOM comparison.

\section{G. Europa Ice Fishing Program Summary}

The Europa Ice Fishing Program example, while fictional, illustrated several of the key principles and products of affordability engineering. The Master Cost WBS was introduced as a tool to compare multiple program alternatives on a common basis. The integrated cost analysis process was summarized, with examples of a sand chart and cumulative cost bar chart for visualization of costs through key program milestones. Sensitivity analysis, enabled through multi-disciplinary analysis frameworks, was discussed as a way to identify cost drivers at the architecture and element level of cost estimating. Finally, quantification of uncertainty through probabilistic costrisk analysis and quantification of the value or benefit associated with various program options through FOM evaluation were presented.

Using these tools and techniques, Flash Gorton was able to explore the program design trade space and evaluate affordability in a holistic manner. Integrated cost analysis was used to characterize the cost composition of several candidate campaigns. Sensitivity analysis identified key cost drivers and could be used to refine the architecture or individual element estimates. It also could help Flash determine where to devote limited resources for further analysis. Through probabilistic analysis of the integrated cost analysis, Flash gained insight about the probability of accomplishing the program within the allotted budget. Armed with this knowledge, Flash Gorton was well on his way to engineering an affordable Europa Ice Fishing Program.

\section{Summary}

Affordability engineering is a complex and multi-disciplinary engineering discipline that resides at the central core of systems engineering and systems analysis. This paper introduces the concept of affordability engineering and explains how it differs from traditional cost estimating and cost methodologies. A formal definition is provided, and seven supporting sub-discipline domains are identified and described. The concepts and methods related to affordability engineering were then illustrated using a fictional manned mission to Jupiter's moon Europa, including a multi-tiered WBS structure that could be applied to any multi-system architecture design, an overview of 
integrated cost estimating, a multidisciplinary design context for affordability engineering, sensitivity analysis for identifying cost drivers and iterating toward affordability, and probabilistic analysis for uncertainty characterization of cost estimates.

The purpose of this paper has been to address a gap in available literature where cost estimating methods have not been extended to adequately assess affordability of multi-system architectures. The concept of affordability as an engineering discipline had also not been formally documented. The definitions and domain frameworks proposed herein were formulated from lessons learned during numerous years supporting government programs with conceptual level affordability integration activities for lunar and Mars exploration applications. It is the authors' suggestion that future research further build upon these affordability principles. There is still much to be researched, and every organization pursuing conceptual studies of complex systems will have its own perspective. It is the authors' hope that this paper provides insight into this complex domain. It is their firm belief that robust, truly affordable, designs will only be achievable when organizations engineer systems with full inclusion of affordability considerations in engineering design trades to reduce costs and manage available resource uncertainties.

\section{References}

${ }^{1}$ Fisher, G.H., Cost Considerations in Systems Analysis, Elsevier Publishing Company, Inc., New York, 1971, Chap. 3.

${ }^{2}$ National Aeronautics and Space Administration, "2008 NASA Cost Estimating Handbook”, Washington, D.C., 2008.

${ }^{3}$ Brady, J., "Systems Engineering and Cost as an Independent Variable", Systems Engineering, Vol. 4, No. 4, 2001, pp. 233241.

${ }^{4}$ Hari, A., Shoval, S., and Kasser, J., "Conceptual Design to Cost: A new systems engineering tool", $18^{\text {th }}$ Annual International Symposium of INCOSE, The Netherlands, June 15-19, 2008.

${ }^{5}$ Dean, E.B., "Cost Reduction vs. Cost Estimating”, NASA 2010 Cost Symposium, Kansas City, Missouri, July 13-15, 2010.

${ }^{6}$ International Council on Systems Engineering, "Systems Engineering Handbook: A Guide for System Life Cycle Processes and Activities", INCOSE-TP-2003-002-03.1, Ver. 3.1, INCOSE, 2007.

${ }^{7}$ Reeves, J.D., Lim, E., “Conceptual Level Affordability Integration”, NASA 2010 Cost Symposium, Kansas City, Missouri, July 13-15, 2010.

${ }^{8}$ DePasquale, D., Charania, A., "I-RaCM: A Fully Integrated Risk and Life Cost Model," AIAA-2008-7703, AIAA Space 2008 Conference and Exposition, San Diego, California, September 9-11, 2008. 